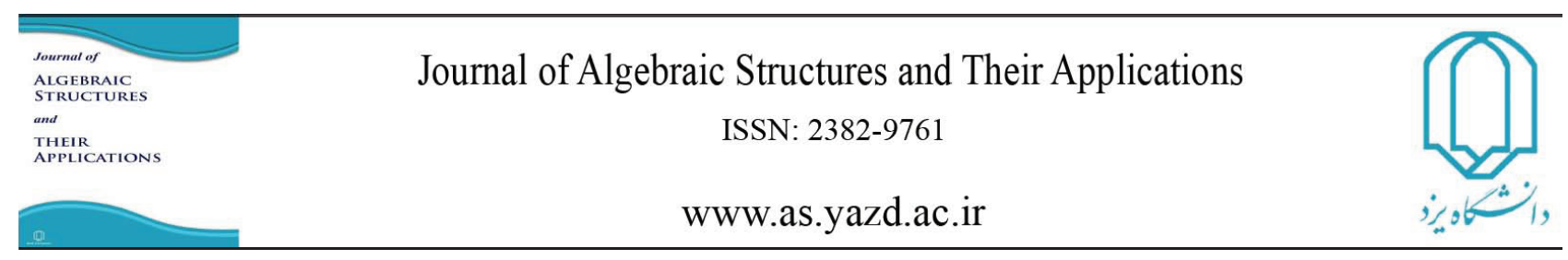

Algebraic Structures and Their Applications Vol. 5 No. 1 ( 2018 ) pp 23-39.

\title{
AN EFFICIENT ALGORITHM FOR MIXED DOMINATION ON GENERALIZED SERIES-PARALLEL GRAPHS
}

\author{
M. RAJAATI, M. R. HOOSHMANDASL* , A. SHAKIBA, P. SHARIFANI AND M. J. DINNEEN
}

\author{
Communicated by S. Alikhani
}

\begin{abstract}
A mixed dominating set $S$ of a graph $G=(V, E)$ is a subset of vertices and edges like $S \subseteq V \cup E$ such that each element $v \in(V \cup E) \backslash S$ is adjacent or incident to at least one element in $S$. The mixed domination number $\gamma_{m}(G)$ of a graph $G$ is the minimum cardinality among all mixed dominating sets in $G$. The problem of finding $\gamma_{m}(G)$ is known to be NP-complete. In this paper, we present an explicit polynomial-time algorithm using the parse tree to construct a mixed dominating set of size $\gamma_{m}(G)$ where $G$ is a generalized series-parallel graph.
\end{abstract}

\section{INTRODUCTION}

A subset of vertices and edges $S \subseteq V \cup E$ in a graph $G=(V, E)$ is a mixed dominating set if for every $v \in(V \cup E) \backslash S, v$ is either adjacent or incident to at least one element in $S$. The mixed domination problem, also known as the total cover problem, is a variant of the classical dominating set problem and was introduced by Alavi et. al in 1977 [2]. Placing phase measurement units(PMUs) in an electric power system is one of its known applications [15]. The mixed domination number of a

DOI:http://dx.doi.org/10.29252/asta.5.1.23

MSC(2010): Primary:05C85

Keywords: Mixed Dominating Set; Generalized Series-Parallel; Parse Tree; Tree-width.

Received: 20 April 2018, Accepted: 01 September 2018.

*Corresponding author

(C) 2018 Yazd University. 
graph $G$ is the minimum cardinality among all mixed dominating sets of $G$ and is denoted by $\gamma_{m}(G)$. In [2], Alavi et. al. showed that for a connected graph $G$ of order $n$, the value of $\gamma_{m}(G)$ is bounded from above by $\lceil n / 2\rceil$. In [3], they have also illustrated some extremal cases and gave some properties for connected graphs, which have a total covering number equal to $\lceil n / 2\rceil$. In [I]], Majumdar showed that the problem of finding $\gamma_{m}(G)$ is NP-complete for general graphs. Also, it is shown that this problem remains $N P$-complete even if it is restricted to chordal graphs [7], planar bipartite graphs [12], and split graphs[10, 15] . Finding a mixed dominating set of minimum cardinality is tractable for some families of graphs such as trees [1, 15, 10], cactus graphs [10] and graphs with bounded tree-width[13].

Rajaati et. al. proposed a dynamic programming algorithm to solve the mixed domination problem on graphs with bounded tree-width using the tree decomposition of graphs in [13]. In this paper, we use the parse tree of graphs to present an explicit polynomial-time algorithm to construct a mixed dominating set for generalized series-parallel graphs in linear time. Moreover, we enumerate the number of $\gamma_{m}$-sets of $G$. The rest of the paper is organized as follows: In Section D, we review some basic definitions and set our notions. In Section B, we present a linear time algorithm to find a $\gamma_{m}$-set and determine the number of $\gamma_{m}$-sets for $G$ using the parse tree of a generalized series-parallel graph $G$. Then, we analyze the correctness and computational complexity of the proposed algorithms.

\section{PRELIMinaries}

In this section, we review some basic requirements on graph theory and set our notations. For notation and terminology which are not listed here, an interested reader is advised to consult [14].

Let $G=(V, E)$ be a graph with vertex set $V$ and edge set $E$. The (open) neighborhood of a vertex $v \in V$ in $G$ is the set of all vertices adjacent to $v$ and is denoted by $N_{G}(v)$. The closed neighborhood of a vertex $v$ in $G$ is defined as $N_{G}[v]=N_{G}(v) \cup\{v\}$. The mixed neighborhood of $r$ in $G$, for an element $r \in V \cup E$, is denoted by $N_{G}^{m d}(r)$ and is defined as $N_{G}^{m d}(r)=\{s \in V \cup E \mid$ $s$ is adjacent or incident to $r$. Similarly, the closed mixed neighborhood of $r$ is denoted by $N_{G}^{m d}[r]$ and is equal to $N_{G}^{m d}[r]=N_{G}^{m d}(r) \cup\{r\}$.

The problem of domination and its variations are well-studied topics in the literature of graph theory [5, 6]. The mixed domination problem is one of these variants. A subset $S \subseteq V \cup E$ is a mixed dominating set if for every $r \in V \cup E$, it is the case that $\left|N_{G}^{m d}[r] \cap S\right| \geq 1$. The minimum cardinality among such sets is denoted by $\gamma_{m}(G)$. Moreover, a $\gamma_{m}$-set for $G$ is a mixed dominating set of size $\gamma_{m}(G)$.

Definition 2.1 (Generalized Series-Parallel Graphs[4]). A generalized series-parallel, or GSP for short, is a graph $G=(V, E, s, t)$ with two distinguished vertices $s, t \in V$, which are called terminals, and is defined recursively as follows:

(1) $o_{i}$ : A graph $G$ consisting of two vertices connected by a single edge is a $G S P$. 
(2) $o_{s}$ : Given two GSP graphs $G_{1}=\left(V_{1}, E_{1}, s_{1}, t_{1}\right)$ and $G_{2}=\left(V_{2}, E_{2}, s_{2}, t_{2}\right)$, the series operation of $G_{1}$ and $G_{2}$ is denoted by $G_{1} o_{s} G_{2}$ and is a new $G S P$ graph $G=\left(V, E, s_{1}, t_{2}\right)$ where

$$
V=\left(V_{1} \cup V_{2}\right) \backslash\left\{s_{2}\right\},
$$

and

$$
E=\left(E_{1} \cup E_{2} \cup\left\{\left\{t_{1}, v\right\}: v \in N_{G_{2}}\left(s_{2}\right)\right\}\right) \backslash\left\{\left\{s_{2}, v\right\}: v \in N_{G_{2}}\left(s_{2}\right)\right\} .
$$

(3) $o_{p}$ : Given two GSP graphs $G_{1}=\left(V_{1}, E_{1}, s_{1}, t_{1}\right)$ and $G_{2}=\left(V_{2}, E_{2}, s_{2}, t_{2}\right)$, the parallel operation of $G_{1}$ and $G_{2}$ is denoted by $G_{1} o_{p} G_{2}$ and is a new $G S P$ graph $G_{1} o_{p} G_{2}=\left(V, E, s_{1}, t_{1}\right)$ where

$$
V=\left(V_{1} \cup V_{2}\right) \backslash\left\{s_{2}, t_{2}\right\}
$$

and

$$
\begin{array}{r}
E=\left(E_{1} \cup E_{2} \cup\left\{\left\{s_{1}, v\right\}: v \in N_{G_{2}}\left(s_{2}\right)\right\} \cup\left\{\left\{t_{1}, v\right\}: v \in N_{G_{2}}\left(t_{2}\right)\right\}\right) \backslash \\
\left(\left\{\left\{s_{2}, v\right\}: v \in N_{G_{2}}\left(s_{2}\right)\right\} \cup\left\{\left\{t_{2}, v\right\}: v \in N_{G_{2}}\left(t_{2}\right)\right\}\right)
\end{array}
$$

(4) $o_{g}$ : Given two $G S P$ graphs $G_{1}=\left(V_{1}, E_{1}, s_{1}, t_{1}\right)$ and $G_{2}=\left(V_{2}, E_{2}, s_{2}, t_{2}\right)$, the generalized series operation of $G_{1}$ and $G_{2}$ is denoted by $G_{1} o_{g} G_{2}$ and is a new $G S P$ graph $G_{1} o_{g} G_{2}=$ $\left(V, E, s_{1}, t_{1}\right)$ where

$$
V=\left(V_{1} \cup V_{2}\right) \backslash\left\{s_{2}\right\}
$$

and

$$
E=\left(E_{1} \cup E_{2} \cup\left\{\left\{t_{1}, v\right\}: v \in N_{G_{2}}\left(s_{2}\right)\right\}\right) \backslash\left(\left\{\left\{s_{2}, v\right\}: v \in N_{G_{2}}\left(s_{2}\right)\right\}\right) .
$$

Any $G S P$ graph is obtained by finite applications of rules (1) through (4).

If the rule (4) is removed, then we obtain a subclass of GPSs called series-parallel or $S P$ graphs. These rules are illustrated in Figure $\mathbb{W}$. Note that in Figure $\mathbb{W}$, the graph $\left(\widehat{G} o_{g}\left(G_{1} o_{s} G_{2}\right)\right) o_{s}\left(\left(G_{1} o_{s} G_{2}\right) o_{p} \widehat{G}\right)$ is a $G S P$, however it is not an $S P$. We generalize the concept
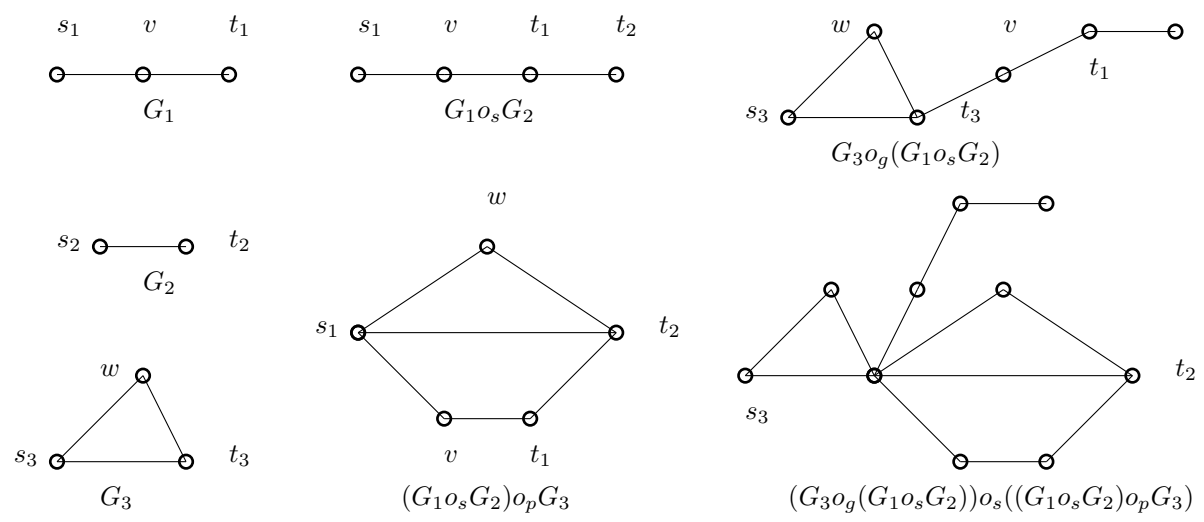

FIGURE 1. An illustration of applying GSP rules. 
of $p$-graph, which are defined for $S P \mathrm{~s}$ in [9], for $G S P$ graphs in the following definition.

Definition 2.2 ( $p$-graph). Let $G=(V, E, x, y)$ be a GSP and $\widehat{G}=(\widehat{V}, \widehat{E}, \widehat{x}, \widehat{y})$ be a subgraph of $G$ satisfying the following conditions:

(1) either $\widehat{x}=x$ or $x \notin \widehat{V}$ and there exists an edge $\{u, v\} \in E \backslash \widehat{E}$ such that $v=\widehat{x} \in \widehat{V}$.

(2) either $\widehat{y}=y$ or $w \notin \widehat{V}$ and there exists an edge $\{w, z\} \in E \backslash \widehat{E}$ such that $z=\widehat{y} \in \widehat{V}$.

Then, $\widehat{G}$ is called a $p$-graph of $G$.

A generalized series-parallel graph $G$ can be represented by a binary parse tree $T$ which is defined as follows.

Definition 2.3 (Binary Parse Tree for $G S P$ Graphs[9]). A binary parse tree for $G S P$ graph $G$ is defined recursively as follows:

(1) A tree consisted of a single vertex labeled $(u, v)_{i}$ is a binary parse tree for primitive $G S P$, $G=(\{u, v\},\{u, v\}, u, v)$.

(2) Let $G=(V, E)$ be a $G S P$ obtained by some composition of two other $G S P$ graphs $G_{1}$ and $G_{2}$, and $T_{1}$ and $T_{2}$ be their binary parse trees, respectively. Then, a binary parse tree for $G$ is a tree with the root $r$ labeled as either $(u, v)_{s},(u, v)_{p}$ or $(u, v)_{g}$ depending on which operation is used to generate $G$. The vertices $u$ and $v$ are terminals of $G$ and the roots of $T_{1}$ and $T_{2}$ are the left and the right children of $r$, respectively.

It is obvious that in any binary parse tree for a $G S P$ graph $G$, every internal vertex of the tree has exactly two children and there are $|E|$ leaves.

Remark 2.4. Note that when we use a label $(x, y)$, we do not care about the label being either $(x, y)_{i},(x, y)_{s},(x, y)_{p}$ and $(x, y)_{g}$.

Let $t$ be an internal vertex of a binary parse tree $T$, and for $G S P$ graph $G, \tau(t)$ denote the subtree of $T$ rooted at $t$. Also, the left and the right subtrees of $t$ are denoted by $\tau_{\ell}(t)$ and $\tau_{r}(t)$, respectively. Then, the vertices of $T$ are labeled as follows:

(a) For each edge $e=\{x, y\} \in E$, there exists exactly one leaf which is labeled by $(x, y)$ in $T$.

(b) For each internal vertex $t \in V_{T}$, which is labeled by $(x, y)_{s}$, the root of $\tau_{\ell}(t)$ is labeled by $(x, z)$ and the root of $\tau_{r}(t)$ is labeled by $(z, y)$ where $z$ is some vertex in $V$. These vertices are called $s$-vertices.

(c) For each internal vertex $t \in V_{T}$ labeled $(x, y)_{p}$, the root of $\tau_{\ell}(t)$ and $\tau_{r}(t)$ are labeled by $(x, y)$. These vertices are called $p$-vertices.

(d) For each internal vertex $t \in V_{T}$ labeled $(x, z)_{g}$, the root of $\tau_{\ell}(t)$ is labeled by $(x, z)$ and the root of $\tau_{r}(t)$ is labeled by $(z, y)$ where $z$ is a vertex in $V$. These vertices are called $g$-vertices. 
A binary parse tree for a $G S P$ graph $G$ is illustrated in Figure 2 . Note that there may exist several binary parse trees for a $G S P$ and the binary parse tree is not necessarily unique. Given a $G S P$ graph $G$, a binary parse tree is computable by a linear time algorithm according to the following Lemma.

Lemma 2.5 ([8]). For a given GSP graph $G$, a binary parse tree can be found in linear time.

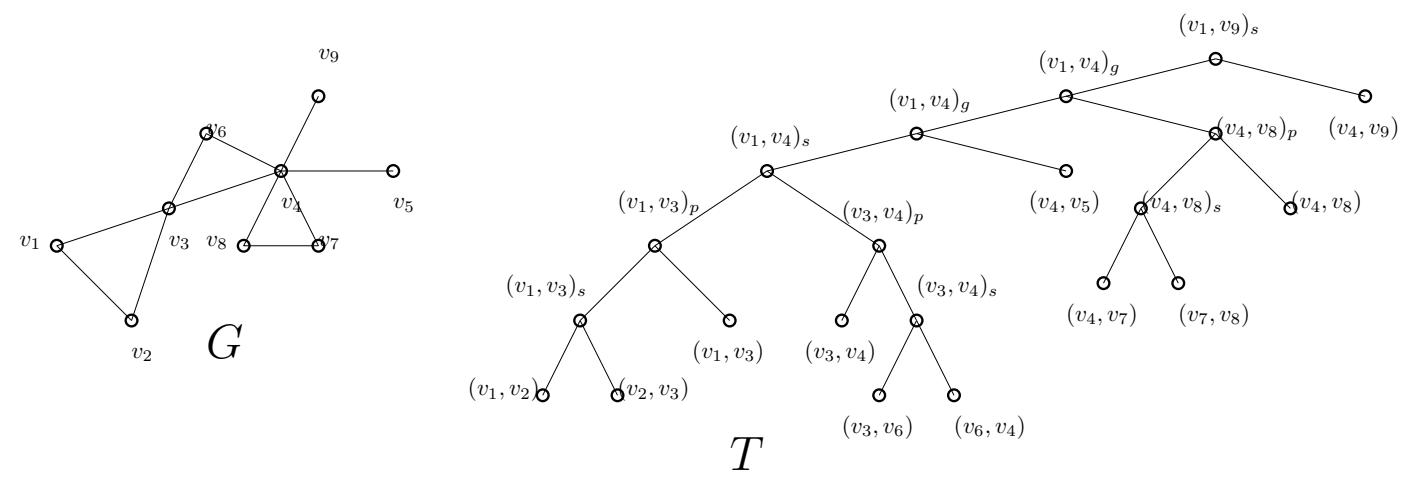

FIgURE 2. A binary parse tree for a $G S P$ graph $G$.

\section{A DYNAMIC PROGRAMMING ALGORITHM TO FIND A MINIMUM MIXED DOMINATING SET}

In this section, we first set some necessary notations which are used throughout the section. Then, we present our proposed algorithm in details to find a $\gamma_{m}$-set, count them and computing $\gamma_{m}(G)$ for a given $G S P$ graph $G$.

Let $t$ be a vertex in a parse tree $T$, for a $G S P$ graph $G$, and $\widehat{G}$ be a $p$-graph of subtree with root $t$.We define the sets $\operatorname{ch}(t)$ and $\mathcal{M M D}_{i, j}(x, y)$ as follow:

- The set $c h(t)$ consists of all children of $t$. In other words, in a parse tree $T$, if $t$ is a leaf vertex, then $c h(t)$ is an empty set and if it is an internal node, then $c h(t)$ contains two elements.

- Let $(x, y)$ be the label of $t$ and $i, j \in\{0,1,2,3,4,5,6\}$. The set $\mathcal{M M D}_{i, j}(x, y)$ is a $\gamma_{m}$-set for $\widehat{G}$ where the label of the vertex $t$ is $(x, y)$, and $i, j$ satisfy one of the following conditions:

Case 0.: If $i=0$, then $x \in \mathcal{M M D} \mathcal{D}_{i, j}(x, y)$ and at least one of its incident edges like $e$ which belongs to $\mathcal{M M D}_{i, j}(x, y)$.

Case 1.: If $i=1$, then $x \in \mathcal{M M D}_{i, j}(x, y)$ and none of its incident edges are in $\mathcal{M M D}_{i, j}(x, y)$.

Case 2.: If $i=2$, then $x \notin \mathcal{M M \mathcal { D }} \mathcal{D}_{i, j}(x, y)$ and at least one of its incident edges like $e$ are in $\mathcal{M M D}_{i, j}(x, y)$.

Case 3.: If $i=3$, then $x \notin \mathcal{M M D}_{i, j}(x, y)$ and none of its incident edges are in $\mathcal{M M D}_{i, j}(x, y)$, since all of them are dominated by an edge or a vertex in $\mathcal{M M D}_{i, j}(x, y)$. Moreover, there is a vertex like $x^{\prime}$ in $\mathcal{M M D}_{i, j}(x, y)$ such that $\left\{x, x^{\prime}\right\} \in E(\widehat{G})$. 
Case 4.: If $i=4$, then $x \notin \mathcal{M M \mathcal { M }} \mathcal{D}_{i, j}(x, y)$ and at least one of its incident edges are not dominated. Moreover, there is a vertex like $x^{\prime}$ in $\mathcal{M M D}_{i, j}(x, y)$ such that $\left\{x, x^{\prime}\right\} \in$ $E(\widehat{G})$.

Case 5.: If $i=5$, then $x \notin \mathcal{M M \mathcal { M }} \mathcal{D}_{i, j}(x, y)$ and none of its incident edges are in $\mathcal{M M D}_{i, j}(x, y)$, since all of them are dominated by an edge or a vertex in $\mathcal{M M D}_{i, j}(x, y)$. Moreover, there is no vertex like $x^{\prime}$ in $\mathcal{M M D}_{i, j}(x, y)$ such that $\left\{x, x^{\prime}\right\} \in E(\widehat{G})$.

Case 6.: If $i=6$, then $x \notin \mathcal{M M D}_{i, j}(x, y)$, none of its incident edges are in $\mathcal{M M D}_{i, j}(x, y)$, and at least one of them is not dominated. Moreover, there is no vertex like $x^{\prime}$ in $\mathcal{M M D}_{i, j}(x, y)$ such that $\left\{x, x^{\prime}\right\} \in E(\widehat{G})$.

These cases can be defined for $y$ based on $j$, similarly. We use Minsize $(\cdots)$ to denote a set with smallest cardinality among the input sets. With these definitions, our proposed algorithm constructs $\mathcal{M M D}_{i, j}(x, y)$ as a mixed dominating set of minimum cardinality for graph $G(x, y)$. It also computes $\mathcal{N}_{i, j}(x, y)$ as the number of minimal cardinality mixed dominating sets.

Now, we are ready to state our algorithm. The algorithm finds a binary parse tree like $T$ for the input $G S P$ graph $G$ in linear time using the procedure described in [8]. Next, it traverses $T$ in a bottom-up order. Each subtree in the parse tree corresponds to a $p$-graph for $G$ and each vertex $t$ of $T$ is labeled by either $(x, y)_{i},(x, y)_{s},(x, y)_{p}$ or $(x, y)_{g}$ where $x$ and $y$ are terminals in the corresponding $p$-graph of $\tau(t)$.

For each visiting vertex $t$, one of the procedures Processleaf, ProcessSvertex, ProCessPvertex or ProcessGvertex is called based on the type of $t$. For each procedure, the input is consisted of vertices $x$ and $y$. By traversing the parse tree $T$ and calling proper procedures for each vertex, our algorithm finds a subset of $\mathcal{M M D}_{i, j}(x, y) \subseteq V(\widehat{G})$ where for each $i, j \in\{0,1,2,3,4,5,6\}$, the set $\mathcal{M M D}_{i, j}(x, y)$ stores a minimum mixed dominating set of $\widehat{G}$ with the assumption that $x, y$ or some of their incident edges cannot be dominated.

After visiting the root node of $T$ and computing $\mathcal{M M D}_{i, j}(x, y)$ for it, a $\gamma_{m}$-set for $G$ can be found. Finally, a $\mathcal{M M D}_{i, j}(x, y)$ set with minimum cardinality is returned where $i, j \in\{0,1,2,3\}$.

The input to the ProcessLeaf procedure is a leaf vertex $v \in V_{T}$ labeled by $(x, y)_{l e a f}$, and the its output is a set $\mathcal{M M D}_{i, j}(x, y)$ for $i, j \in \mathcal{M}$. Note that a leaf corresponds to an edge $\{x, y\}$ in $G$. All valid cases for different $i, j \in \mathcal{M}$ can be summarized as follows:

(1) The vertices $x$ and $y$ and the edge $\{x, y\}$ are dominated and at least one of them is a member of $\mathcal{M M D}_{i, j}(x, y)$. So $i, j$ satisfies one of the following conditions:

- $i=0$ and $j \in\{0,2\}$,

- $i=1$ and $j \in\{1,3\}$,

- $i=2$ and $j \in\{0,2\}$,

- $i=3$ and $j=1$. 
(2) The vertices $x$ and $y$ and the edge $\{x, y\}$ are not dominated and are not members of $\mathcal{M M D}_{i, j}(x, y)$. So, we have $i=j=6$.

Let $v$ be a vertex of $T$ labeled by $(x, y)_{s}$. In the ProcessS verTex procedure, we compute the set $\mathcal{M M D}_{i, j}(x, y)$ for given terminal vertices $x, y$ and a common vertex $z$. The sets $\mathcal{M M D}_{\mathcal{D}_{\ell}, j_{\ell}}(x, z)$ and $\mathcal{M M D}_{i_{r}, j_{r}}^{r}(z, y)$ are corresponding to $\tau_{\ell}(t)$ and $\tau_{r}(t)$, where the roots of $\tau_{\ell}(t)$ and $\tau_{r}(t)$ are labeled by $(x, z)$ and $(z, y)$, respectively. The members of $\mathcal{M M D}_{i_{\ell}, j_{\ell}}^{\ell}(x, z), \mathcal{M M D}_{i_{r}, j_{r}}^{r}(z, y)$, and $M M D_{i, j}(x, y)$ are those vertices of $T$ which are corresponding to $p$-graphs $G_{1}=\left(V_{1}, E_{1}, x, z\right)$, $G_{2}=\left(V_{2}, E_{2}, z, y\right)$ and $\widehat{G}=G_{1} o_{s} G_{2}=(\widehat{V}, \widehat{E}, x, y)$, respectively.

The possible cases based on belonging $z$ to $\mathcal{M M D}_{i, j}(x, y)$ and the vertex or edge dominating $z$, are summarized in Table $\mathbb{W}$. To be precise, consider the following cases:

Case 0.: Vertex $z$ and at least one of its incident edges belong to $\mathcal{M M D}_{i, j}(x, y)$. So, we have $\left(j_{\ell}, i_{r}\right) \in\{(0,0),(0,1),(1,0)\}$.

Case 1.: Vertex $z \in \mathcal{M M D}_{i, j}(x, y)$ and none of its incident edges belong to $\mathcal{M M D}_{i, j}(x, y)$ which implies $j_{\ell}=i_{r}=1$.

Case 2.: Vertex $z \notin \mathcal{M M D} \mathcal{D}_{i, j}(x, y)$ and an edge incident to $z$ belong to $\mathcal{M M D}_{i, j}(x, y)$. So, we have either $\left(j_{\ell}, i_{r}\right) \in\{2\} \times\{2,3,4,5,6\}$, or $\left.\left(i_{r}, j_{\ell}\right) \in\{2\} \times\{2,3,4,5,6\}\right)$.

Case 3.: Vertex $z$ and its incident edges does not belong to $\mathcal{M M D}_{i, j}(x, y)$. So, we have $\left(j_{\ell}, i_{r}\right) \in\{(3,3),(3,5),(5,3)\}$.

Now, let $v$ be a vertex of $T$ labeled by $(x, y)_{p}$. The sets $\mathcal{M M D}_{i_{\ell}, j_{\ell}}^{\ell}(x, y)$ and $\mathcal{M M D} \mathcal{M D}_{i_{r}, j_{r}}^{r}(x, y)$ correspond to $\tau_{\ell}(t)$ and $\tau_{r}(t)$, respectively. For each $i, j \in M$, we describe a method for finding $\mathcal{M M D}_{i, j}(x, y)$. Note that it is enough to find a relation among the values $(i, j),\left(i_{\ell}, j_{\ell}\right)$ and $\left(i_{r}, j_{r}\right)$. To do so, we use the procedure FINDLIST. Let the input to this procedure be a value like $i \in M$. Then, the procedure returns a set of pairs which are proper values for $i_{\ell}$ and $i_{r}$. Note that for $j \in M$, the procedure returns proper $j_{\ell}$ and $j_{r}$, similarly.

Note that $\tau_{\ell}(t), \tau_{r}(t)$ and $\tau(t)$ correspond to $p$-graphs $G_{1}=\left(V_{1}, E_{1}, x, y\right), G_{2}=\left(V_{2}, E_{2}, x, y\right)$ and $\widehat{G}=G_{1} o_{p} G_{2}=(\widehat{V}, \widehat{E}, x, y)$, respectively. For $i \in \mathcal{M}$ (resp. $j \in \mathcal{M}$ ), the values of $i_{\ell}$ and $i_{r}$ (resp. $j_{\ell}$ and $j_{r}$ ) are determined as follows. The following cases are also illustrated in Table $\rrbracket$.

Case 0.: $i=0$ implies $\left(i_{\ell}, i_{r}\right) \in\{(0,0),(0,1),(1,0)\}$,

Case 1.: $i=1$ implies $i_{\ell}=i_{r}=1$,

Case 2.: $i=2$ implies either $\left(i_{\ell}, i_{r}\right) \in\{2\} \times\{2,3,4,5,6\}$ or $\left(i_{\ell}, i_{r}\right) \in\{2,3,4,5,6\} \times\{2\}$,

Case 3.: $i=3$ implies $\left(i_{\ell}, i_{r}\right) \in\{(3,3),(3,5),(5,3)\}$,

Case 4.: $i=4$ implies either $\left(i_{\ell}, i_{r}\right) \in\{(3,4),(3,6),(4,4),(4,5),(4,6)\}$ or

$$
\left(i_{r}, i_{\ell}\right) \in\{(3,4),(3,6),(4,4),(4,5),(4,6)\},
$$

Case 5.: $i=5$ implies $i_{\ell}=i_{r}=5$,

Case 6.: $i=6$ implies $\left(i_{\ell}, i_{r}\right) \in\{(5,6),(6,5),(6,6)\}$. 


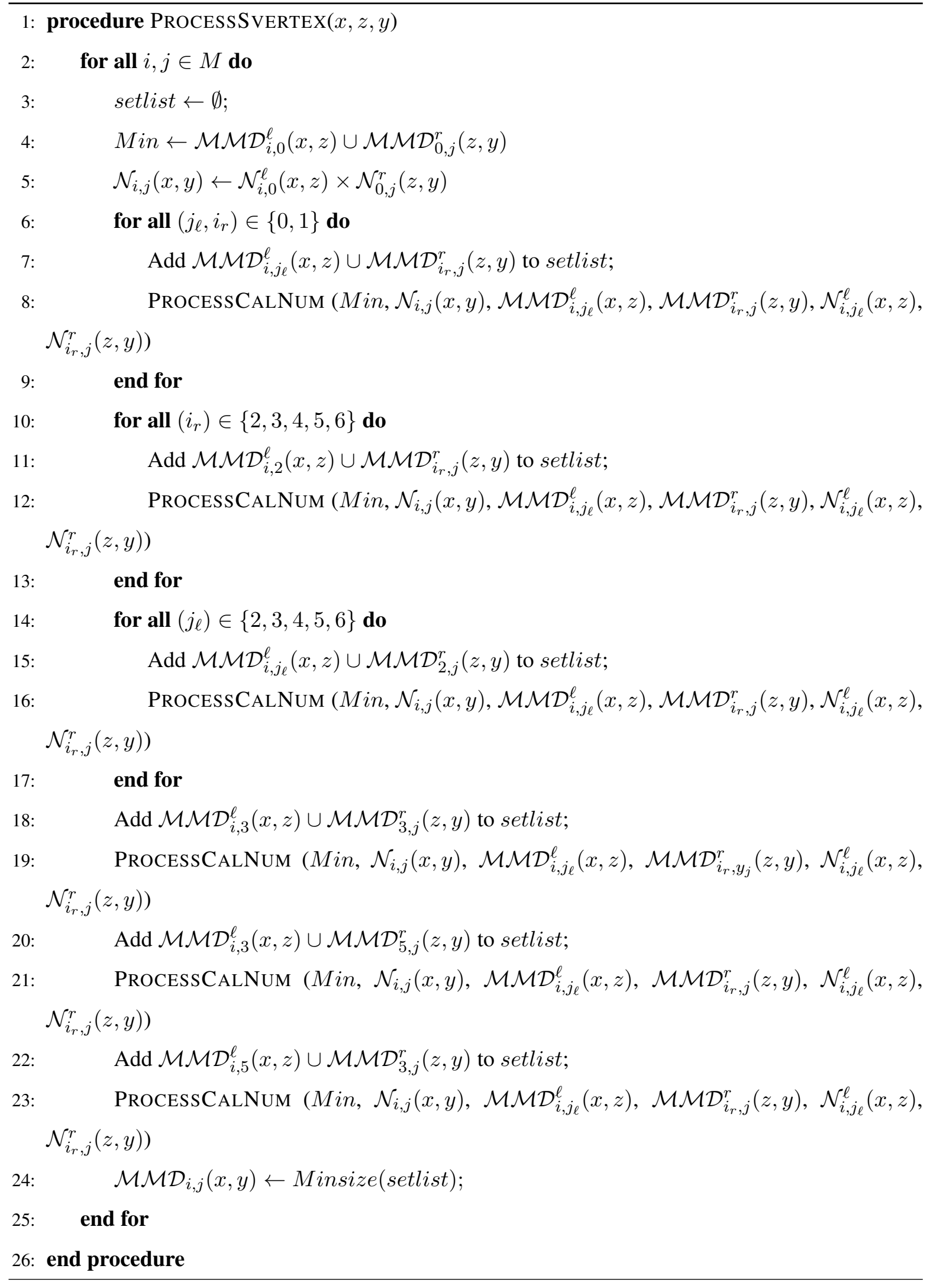




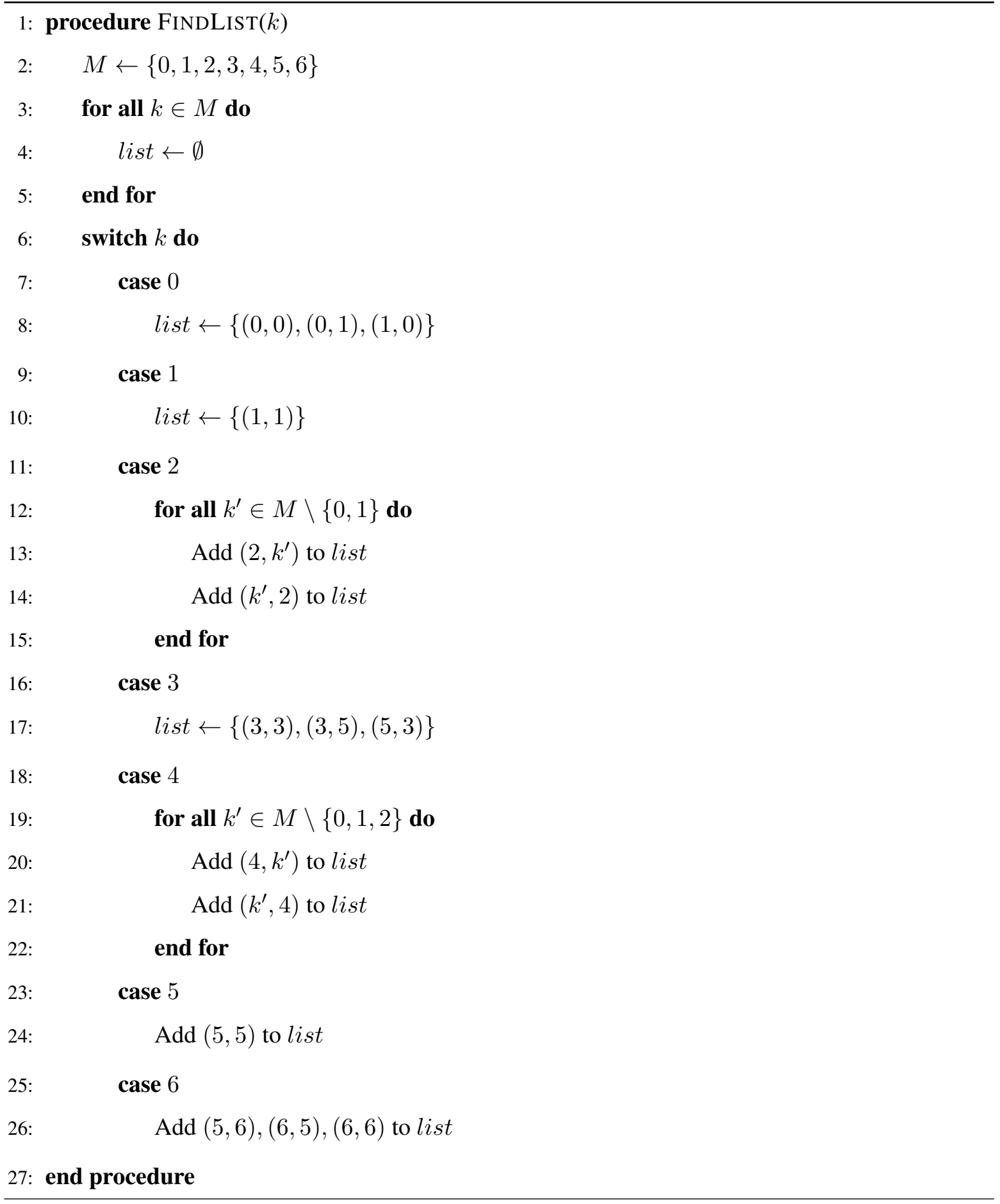

Let $v$ be a vertex of $T$ labeled $(x, y)_{g}$. In the procedure ProcessGvertex, the set $\mathcal{M M D}_{i, j}(x, y)$ is computed for the given vertices $x$ and $y$. The sets $\mathcal{M M D}_{i, j_{\ell}}^{\ell}(x, y)$ and $\mathcal{M M D}_{i_{i_{r}, j_{r}}^{r}}(x, y)$ correspond to $\tau_{\ell}(t)$ and $\tau_{r}(t)$, respectively.

Let the roots of $\tau_{\ell}(t)$ and $\tau_{r}(t)$ be labeled by $(x, y)$ and $(y, z)$, respectively, for some $z \in V$. Also, assume that $\mathcal{M M D}_{i_{\ell}, j_{\ell}}^{\ell}(x, y)$ and $\mathcal{M M D}^{i_{i_{r}, j_{r}}}(x, y)$ are the associated sets with the vertices $(x, y)$ and 

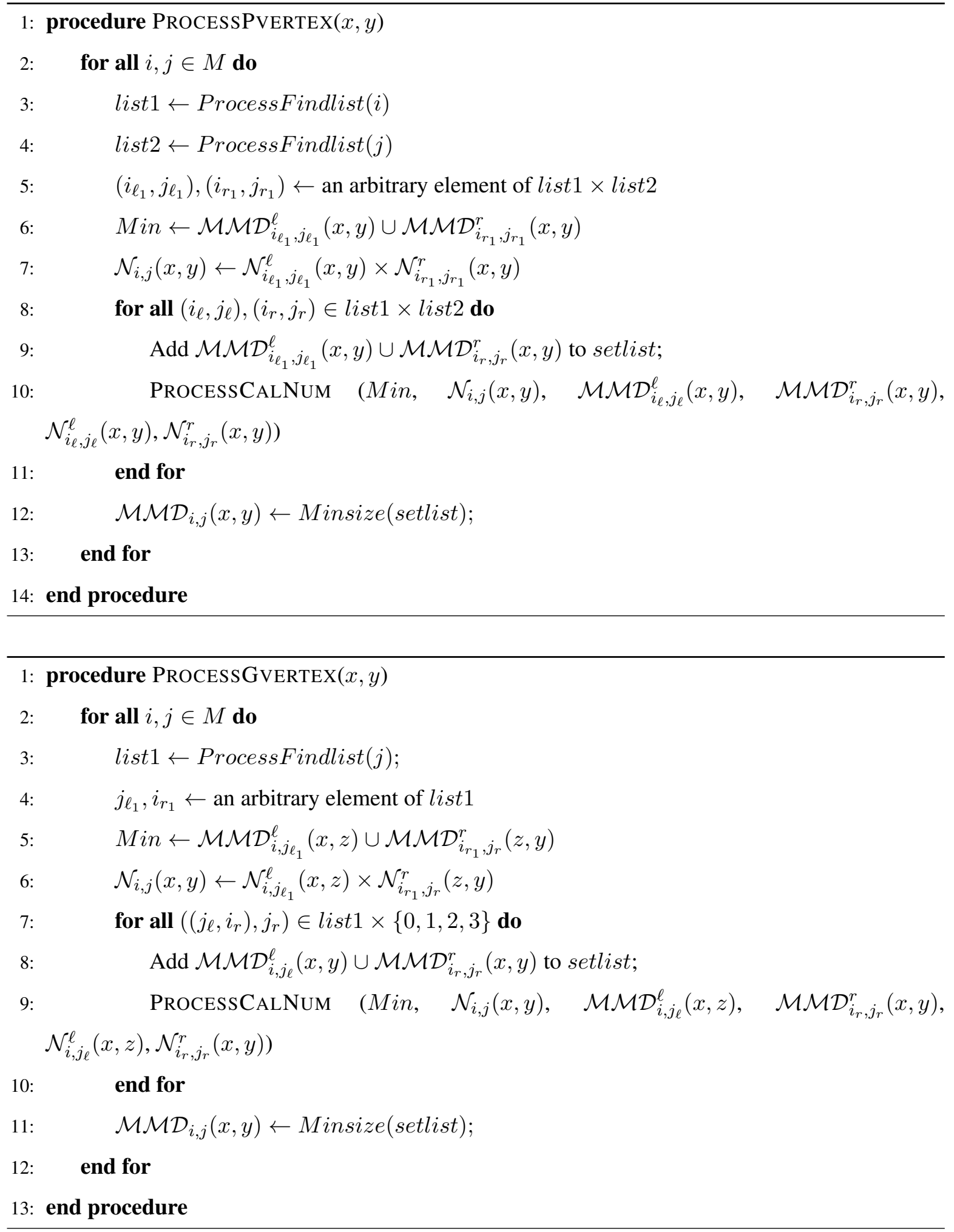

$(y, z)$ of $T$. It is obvious that $z$ does not appear in ancestors of $t$ in the parse tree. So, $z$ and all of its incident edges must be closely dominated which implies $j \in\{0,1,2,3\}$. Since $y$ is the common vertex between $G_{1}$ and $G_{2}$, based on $j$, the set denoted by list 1 , which equals the set of possible pairs, can be 
computed for $j_{\ell}$ and $i_{r}$ by procedure FINDLIST. Several cases are possible for $y$ which are shown in Table 3 which are discussed below:

Case 0.: $j=0$ implies $\left(j_{\ell}, i_{r}\right) \in\{(0,0),(1,0),(0,1)\}$,

Case 1.: $j=1$ implies $j_{\ell}=i_{r}=1$,

Case 2.: $j=2$ implies either $\left(j_{\ell}, i_{r}\right) \in\{2\} \times\{2,3,4,5,6\}$ or $\left(j_{\ell}, i_{r}\right) \in\{2,3,4,5,6\} \times\{2\}$,

Case 3.: $j=3$ implies $\left(j_{\ell}, i_{r}\right) \in\{(3,3),(3,5),(5,3)\}$,

Case 4.: $j=4$ implies either $\left(j_{\ell}, i_{r}\right) \in\{3\} \times\{4,6\},\left(j_{\ell}, i_{r}\right) \in\{4\} \times\{4,5,6\},\left(i_{r}, j_{\ell}\right) \in$ $\{3\} \times\{4,6\}$ or $\left(i_{r}, j_{\ell}\right) \in\{4\} \times\{4,5,6\}$,

Case 5.: $j=5$ implies $j_{\ell}=i_{r}=5$,

Case 6.: $j=6$ implies $\left(j_{\ell}, i_{r}\right) \in\{(5,6),(6,5),(6,6)\}$.

By $\mathcal{M M D}_{i, j}(x, y) \leftarrow \operatorname{MinsizE}($ Setlist $)$, we remove all undefinable sets from Setlist. If Setlist is empty, then $\mathcal{M M D}_{i, j}(x, y)$ becomes undefinable.

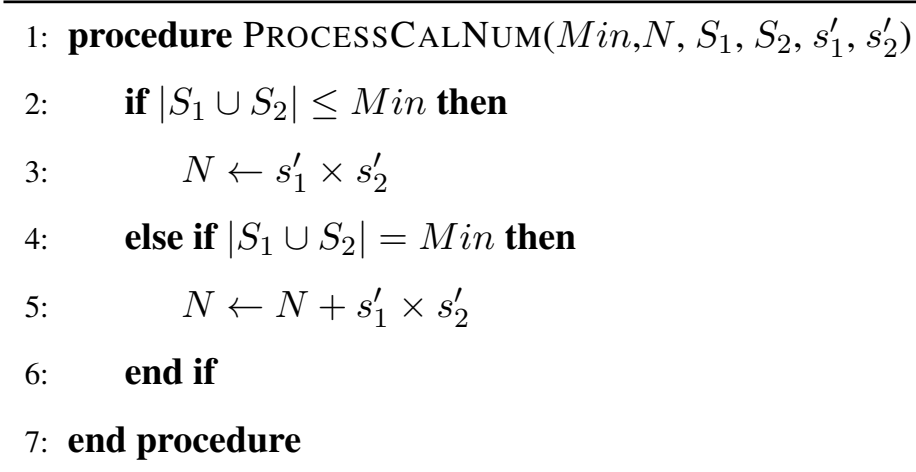

We study the correctness and complexity of our proposed algorithm below.

Theorem 3.1. For a given generalized series-parallel graph $G=(V, E)$, the Algorithm $\square$ finds a $\gamma_{m}$-set for $G$ in time $O(|V|)$.

Proof. In AlgorithmW, we traverse the parse tree $T$ in a bottom-up fashion and compute at most 49 sets for each of its internal vertices. Each initial set for the leaves of the tree represents all possible mixed dominating sets in a graph consisting of only one edge. Let $G_{1}=\left(V_{1}, E_{1}\right)$ and $G_{2}=\left(V_{2}, E_{2}\right)$ be the graphs represented by the subtrees $\tau_{\ell}(t)$ and $\tau_{r}(t)$, respectively. Assume that they are given to the procedures Processleaf, Processsvertex, ProcessPvertex and ProcessGvertex. It is easy to see that these procedures find all possible $\gamma_{m}$-sets in each corresponding graph. Finally, our algorithm extracts only a valid minimum mixed dominating set. The steps of the algorithm require at most $\left|O\left(V_{T}\right)\right|$ operations. Since each binary tree with $n$ leaves has $O(n)$ vertices and the binary parse tree of every $G S P$ graph $G$ has $|E(G)|$ leaves. So, we have $\left|V_{T}\right| \in O(|E(G)|)$. Every $G S P$ graph $G$ is planar. In a planar graph, we have $|E| \leq 3|V|-6$. Also, we know that a parse tree $T$ can be constructed in $O(|V|)[9]$. So, the algorithm computes a $\gamma_{m}$-set for a given GSP graph $G$ in time $O(|V|)$. 


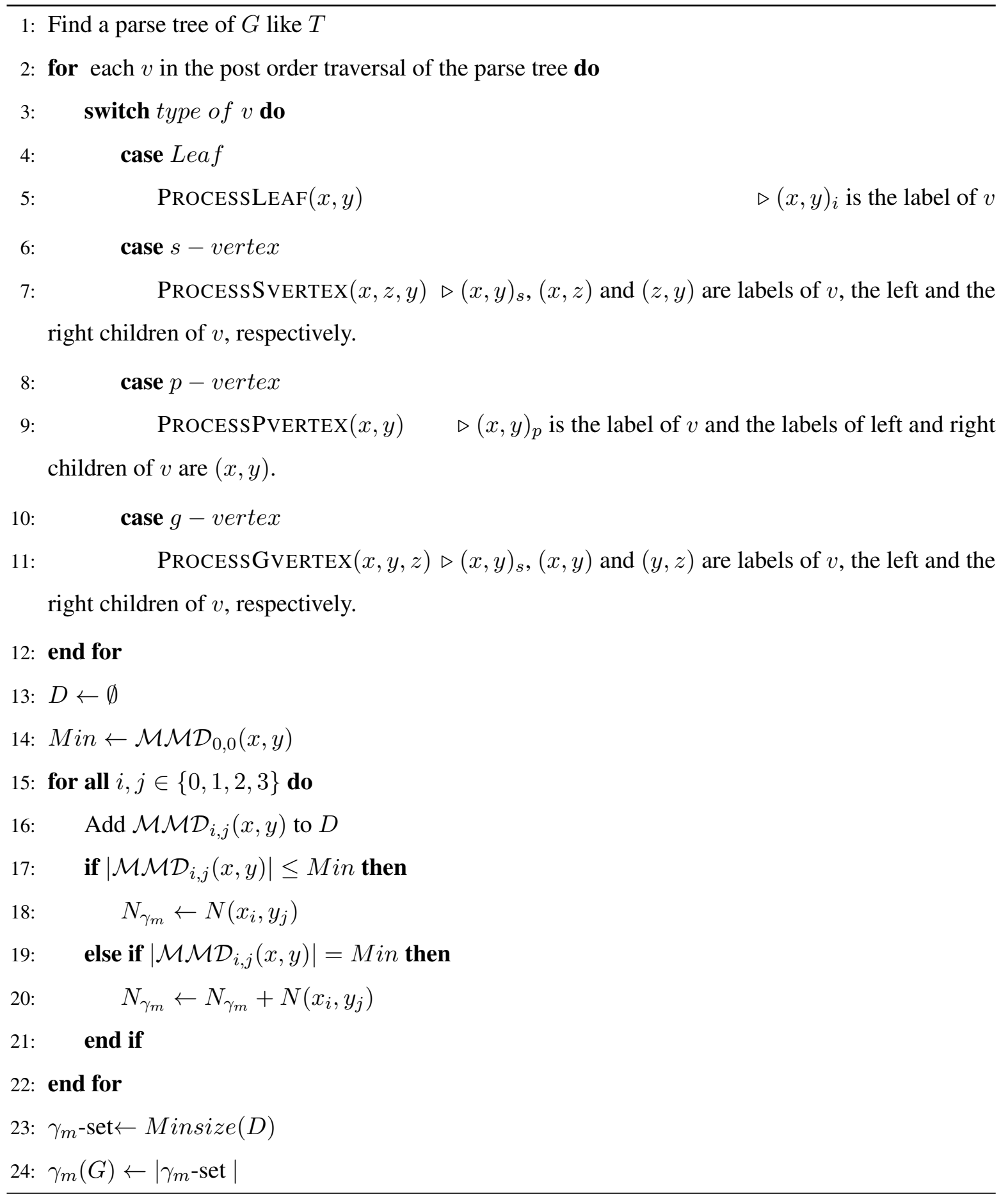

\section{ACKNOWLEDGMENTS}

This article has been written while the fourth author was in a sabbatical visit to University of Auckland. He would like to express his gratitude to Prof. Cristian S. Calude and his research group for the nice and friendly hospitality. 


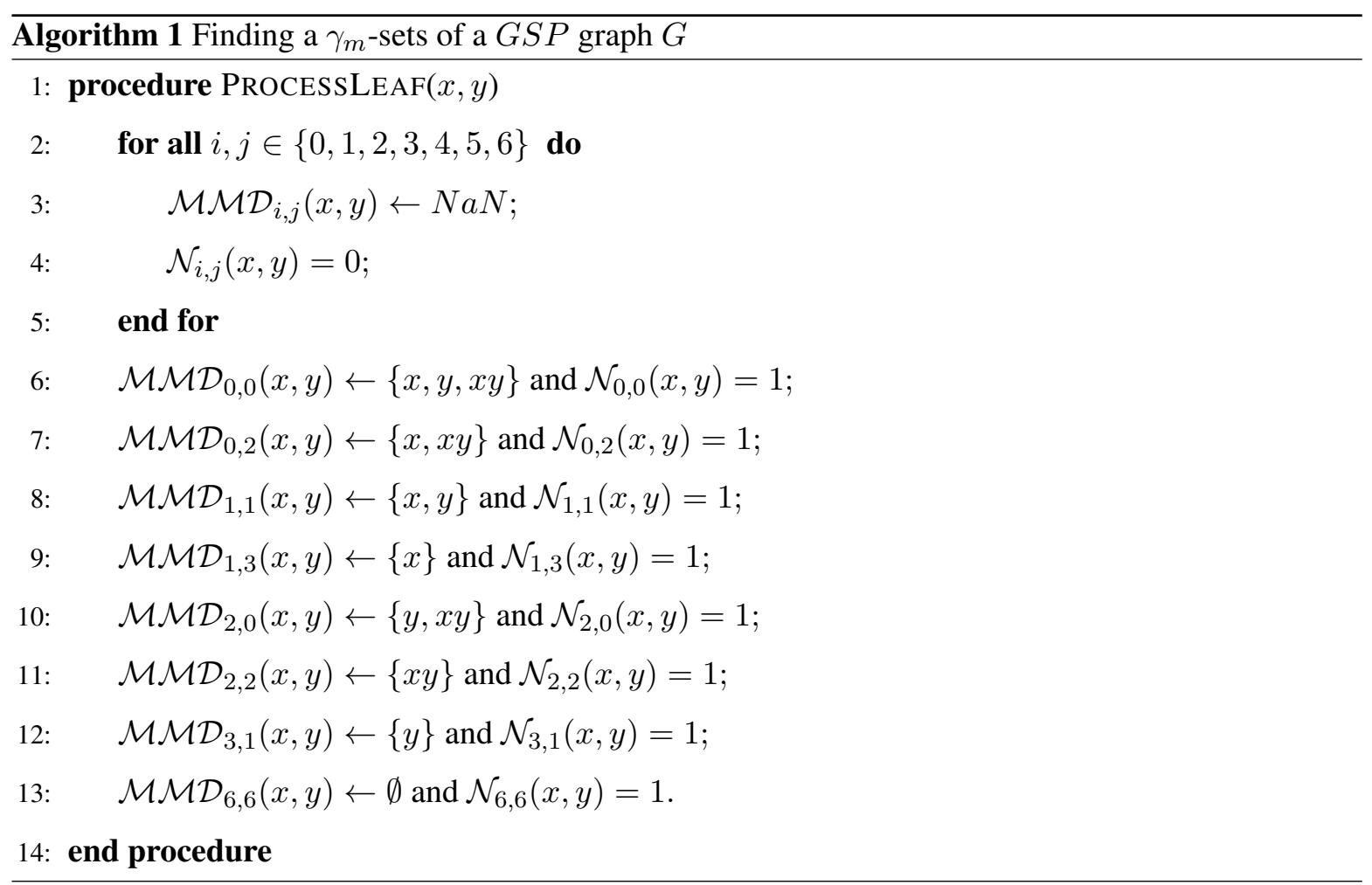

\section{REFERENCES}

[1] G. S. Adhar, S. Peng, Mixed domination in trees: a parallel algorithm, Congr. Numer. 100 (1994) 73-80.

[2] Y. Alavi, M. Behzad, L. M. Lesniak-Foster, E. Nordhaus, Total matchings and total coverings of graphs, J. Graph Theory 1(2) (1977) 135-140.

[3] Y. Alavi, J. Liu, J. Wang, Z. Zhang, On total covers of graphs, Discrete Math. 100 (1992) 229-233.

[4] P. Chebolu, M. Cryan, R. Martin, Exact counting of Euler tours for generalized series-parallel graphs, J. Discrete Algorithms 10 (2012) 110-122.

[5] T. W. Haynes, S. Hedetniemi, P. Slater, Fundamentals of domination in graphs, CRC Press, 1998.

[6] T. W. Haynes, S. Hedetniemi, P. Slater, Domination in graphs: advanced topics, Taylor \& Francis, 1998.

[7] S. M. Hedetniemi, S. T. Hedetniemi, R. Laskar, A. McRae, A. Majumdar, Domination, independence and irredundance in total graphs: a brief survey, Graph Theory, Combinatorics and Applications: Proceedings of the 7th Quadrennial International Conference on the Theory and Applications of Graphs 2 (1995) 671-683.

[8] J. E. Hopcroft, R. E. Tarjan, Dividing a graph into triconnected components, SIAM J. Comput. 2(3) (1973) 135-158.

[9] T. Kikuno, N. Yoshida,Y. Kakuda, A linear algorithm for the domination number of a series-parallel graph, Discrete Appl. Math. 5(3) (1983) 299-311.

[10] J. K. Lan, G. J. Chang, On the mixed domination problem in graphs, Theoret. Comput. Sci. 476(84) (2013) 84-93.

[11] A. Majumdar, Neighborhood Hypergraphs: A Framework for Covering and Packing Parameters in Graphs, PhD thesis, Clemson University, Department of Mathematical Sciences, South Carolina, 1992.

[12] D. F. Manlove, On the algorithmic complexity of twelve covering and independence parameters of graphs, Discrete Appl. Math. 91(1) (1999) 155-175. 
[13] M. Rajaati, M. R. Hooshmandasl, M. J. Dinneen, A. Shakiba, On fixed-parameter tractability of the mixed domination problem for graphs with bounded tree-width, Discrete Math. Theor. Comput. Sci. 20(2) (2018) 1-25.

[14] D. B. West, Introduction to graph theory, Prentice Hall Upper Saddle River, 2001.

[15] Y. Zhao, L. Kang, M. Y. Sohn, The algorithmic complexity of mixed domination in graphs, Theoret. Comput. Sci. 412(22) (2011) 2387-2392.

TABLE 1. Different situations for $s$-vertices

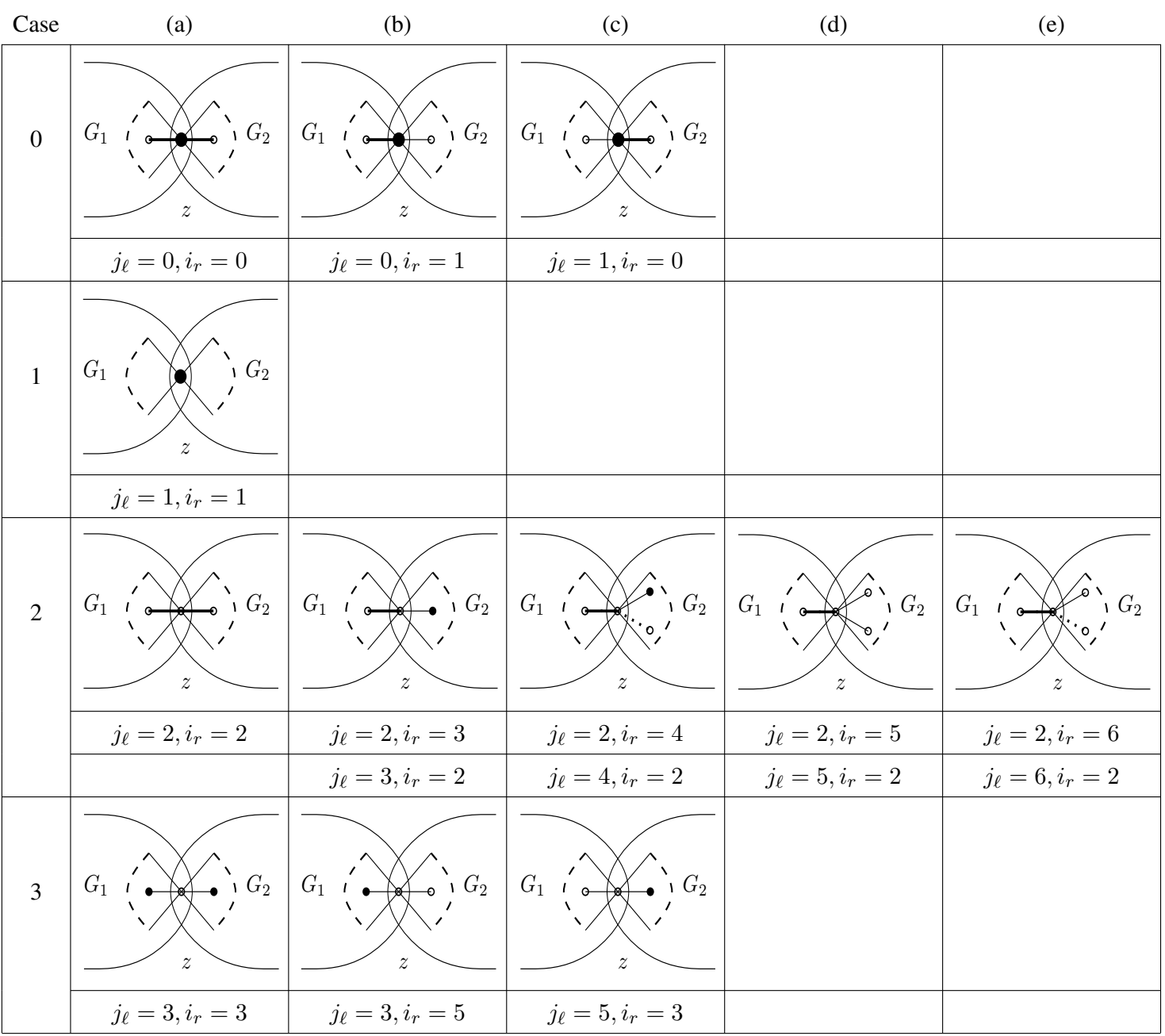


TABLE 2. Different situations for $p$-vertices

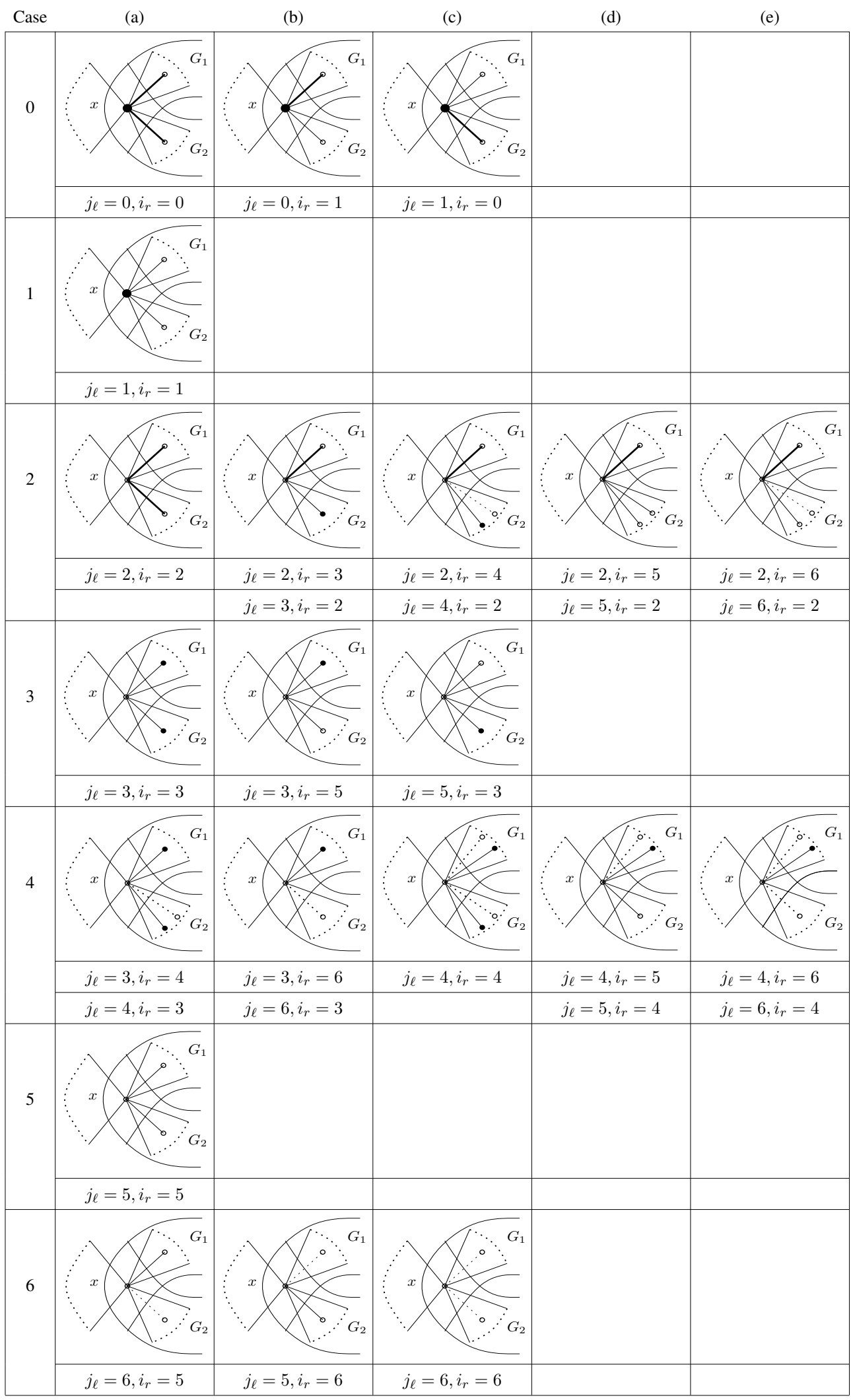


TABLE 3. Different situations for $g$-vertices

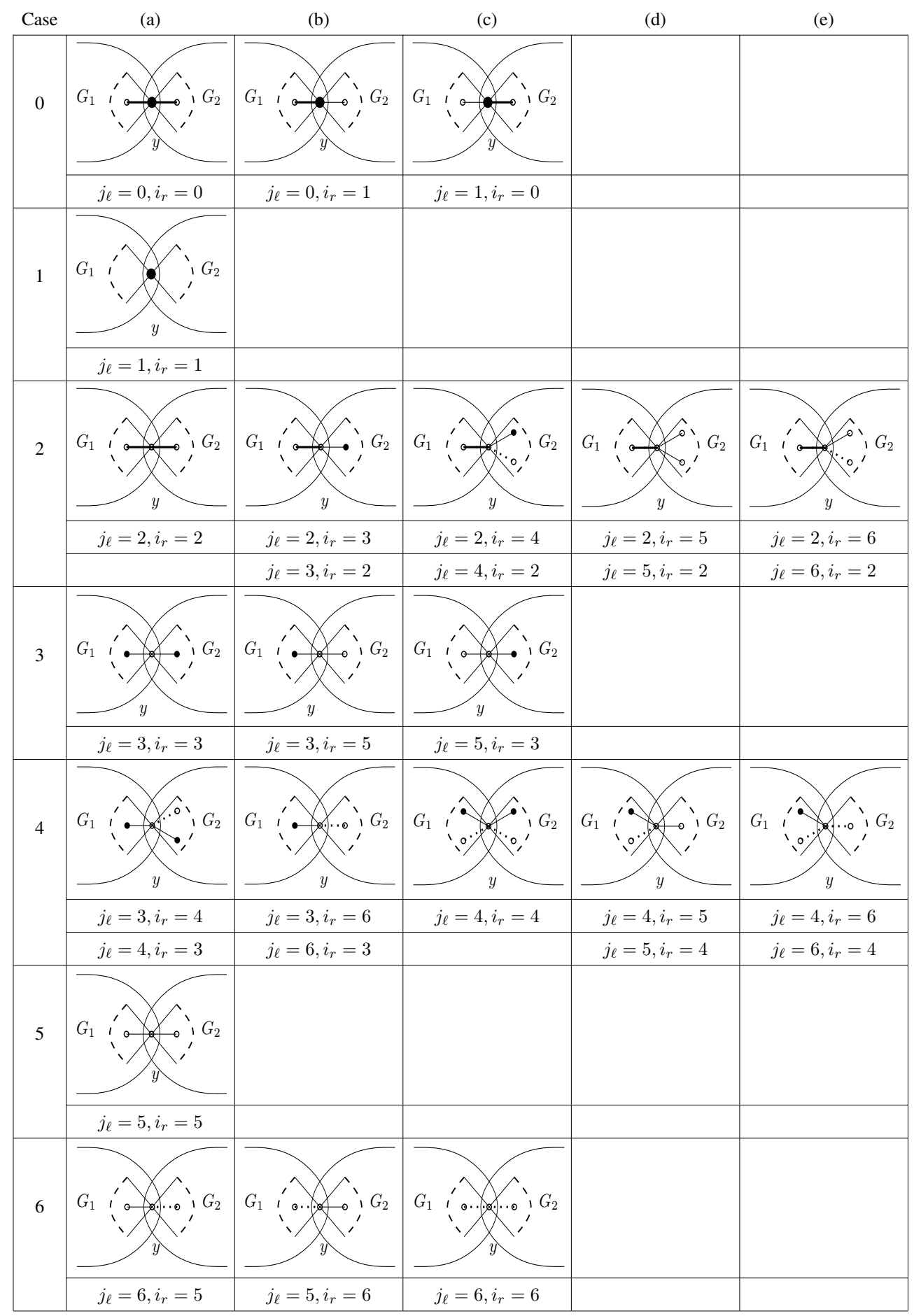

\section{M.Rajaati}

Department of Computer Science

Yazd University 
Alg. Struc. Appl. Vol. 5 No. 1 (2018) 23-39.

Yazd, Iran.

m.rajaati@stu.yazd.ac.ir

\section{R. Hooshmandasl}

Department of Computer Science

Yazd University

Yazd, Iran.

hooshmandasleyazd.ac.ir

A. Shakiba

Department of Computer Science

Vali-e-Asr University of Rafsanjan

Rafsanjan, Iran.

ali.shakibaevru.ac.ir

\section{P. Sharifani}

Department of Computer Science

Yazd University

Yazd, Iran.

pouyeh.sharifaniegmail.com

\section{J. Dinneen}

Department of Computer Science

The University of Auckland

Auckland, New Zealand.

m.dinneen@auckland.ac.nz 